\title{
PrÁCTICAS FILOSÓFICAS EN ITALIA: ORIENTACIONES Y PERSPECTIVAS DESDE SUS ORÍGENES HASTA LA ACTUALIDAD ${ }^{1}$
}

\author{
PHILOSOPHICAL PRACTICES IN ITALY: GUIDELINES AND \\ PERSPECTIVES FROM ITS BEGINNINGS TO THE PRESENT DAY
}

\author{
LUCA NAVE \\ Società Italiana di Counseling Filosofico \\ lucanave1@gmail.com
}

RECIBIDO: 28 DE NOVIEMBRE DE 2015

ACEPTADO: 23 DE JUNIO DE 2015

Resumen: El artículo presenta la historia de la Filosofía Aplicada en Italia desde los años noventa del siglo pasado hasta la actualidad. Se ofrecen reflexiones sobre los principales fundamentos, tratados no sólo en Italia sino a nivel internacional, con el fin de construir los principios epistemológicos y éticos de una filosofía que se convierte en práctica. El ensayo analiza, en particular, la relación entre la Filosofía Aplicada y la Filosofía en la Académica, el mundo "psi" y el arte del counseling, para mostrar las herramientas del de trabajo del orientador filosófico.

Palabras clave: Filosofía Aplicada, filosofía académica, mundo "psi", Counseling, Horizonte socio-político

\begin{abstract}
Italy, starting in the 1990s (nineteen-nineties). We offer some considerations about the main issues addressed, not only in Italy but internationally, in order to build the epistemological and ethical foundations of philosophy as it becomes a practice. In particular, we analyze the relationship between philosophical practice, academic philosophy, the "world of Psy", and the Art of Counseling with the intent to present the utensils in the toolbox of a practical philosopher.
\end{abstract}

Key words: Philosophical practices, Academic philosophy, Psy word, Counseling, Sociopolitical horizon.

\footnotetext{
${ }^{1}$ Traducido al español por Giulia Bazzanella.
} 


\section{Del gueto académico a la vida}

Cada disciplina y cada corriente de pensamiento poseen un punto cero, un momento simbólico desde el cual todo empieza. La corriente internacional de las Practicas Filosóficas nace con la Philosophische Praxis de Gerd Achenbach al inicio de los ochenta. En realidad, desde hace décadas, la filosofía expresaba el deseo de "salir del gueto académico" donde estaba aislada desde siglos atrás y conseguir quedarse con los problemas vividos - no solo los pensados - por las personas de carne y hueso. Todo empieza con la Philosophische Praxis en Alemania: en el año 1982, nace la primera Sociedad internacional para la practica filosófica (Internationale Gesellschaft für Philosophische Praxis, IGPP), que reúne en su interior un numeroso grupo de estudiosos, de múltiple procedencia disciplinar y geográfica, atraídos por el idea de 'practicar' la filosofía.

Al empezar con la Philosophische Praxis, es necesario preguntarse qué comienza exactamente. La idea que de fondo del movimiento completo puede ser resumido de esta manera: sacar la filosofía de las "catedrales del pensamiento" para quedarse con las personas y sus problemas reales, con consultorías individuales o con prácticas filosóficas de grupo con el fin de "hacer" filosofía, utilizar, practicar la filosofía, para decirlo con Platón, "para beneficiar al ser humano". Claro es que esto había de llevarse a cabo de modo distinto a la psicoterapia y, con ello, la Filosofía Aplicada, no se volvería en una simple psicoterapia alternativa.

\section{"El nombre de la cosa"}

La denominación de una disciplina es connotativa: el "nombre" hace referencia a la "cosa", al significado de lo que se hace con la filosofía que vuelve a ser Praxis. El término Praxis en alemán remite a la idea de "practicar", es decir, hacer práctica y operativa, 
la filosofía, y también al "estudio" de un profesional que ofrece un cierto servicio al cliente que lo solicita: un "estudio profesional", como el del notario, el del abogado o del medico. El filósofo se convierte en un consejero, un counselor, un facilitador, un practicante, un orientador, un coach. Su tarea es afín, por varias razones, a la de numerosos expertos de las profesiones de ayuda y de los consejeros presentes en el amplio escenario de la cura de la persona, pero en realidad desempeña un papel esencial, particular y diferente al de todos los demás por provenir de una tradición y manifestar unas competencias peculiares. Ahora bien, ¿a qué tradiciones y competencias estamos aludiendo? Dado que la denominación es connotativa, uno de los primeros problemas con el cual ha sido necesario enfrentarse con miras a la difusión de la disciplina a nivel internacional era la traducción del término Praxis de manera que, fuera de los países de lengua alemana, quedase el mismo "nombre" y se apelase al mismo significado.

Cada disciplina se enfrenta el problema del "nombre de la cosa" y la necesidad de crear un idioma común de modo que el mismo nombre remita al mismo significado en cualquier lugar. El DSM, Manual Diagnostico y Estadístico, surgió de esta necesidad: la de crear un lenguaje internacional común entre los profesionales de la disciplina para que la misma palabra o las mismas expresiones ("depresión", "ataque de pánico" o "ciclotímico") apuntasen a un significado compartido en las distintas partes del mundo.

Primero, con su expansión en Holanda y en los países norteeuropeos y, después, con la llegada en los Estados Unidos, apoyada por el best-seller de Lou Marinoff, Más Platón y menos prozac, la denominación Philosophische Praxis ha sido traducida inicialmente al inglés como Philosophy Practice y, luego, como Philosophical Counseling.

Los problemas semánticos que presenta son claros. El termino Practice recuerda la idea de practicar, de hacer filosofía y no 
destaca el estudio profesional del filósofo; en cambio, Counseling recuerda la practica de la consultoría a la persona y los grupos y connota una práctica y una tradición de pensamiento que no es realmente afín a la Praxis del filosofo. Para paliar a estas complicaciones terminológicas, semánticas y conceptuales, en los diferentes países donde se difundían las Practicas Filosóficas, nacían nombres alternativos como "orientador filosófico", "filósofo practicante", "psico-filosofo", "terapeuta filosofico" y otros, también extravagantes y originales, que evitaremos mencionar. Un marasmo de nombres diferentes que, hoy, necesita una clarificación de lenguaje con el objetivo de mostrar que el filósofo aplicado puede adoptar el apelativo que prefiera siempre y cuando manifieste plena conciencia de que el nombre de la disciplina hace referencia al contexto teórico y a las metodologías que él mismo utiliza en su profesión, es decir, las que hacen referencia al saber y al hacer filosófico de su área de conocimiento. La cuestión del nombre no es sólo terminológica sino ontológica: el nombre conduce a la imagen de lo que se sabe y esto a lo que se sabe hacer con la filosofía en la vida vívidamente. Todo esto requiere claridad conceptual, sentido de responsabilidad y honestad hacia si mismos $\mathrm{y}$ hacia los otros.

El problema del "nombre de la cosa", con todas las problemáticas epistemológicas y éticas anexas y conexas, se presenta en el seno del origen del movimiento de la Practica Filosófica en Italia.

\section{Un discurso sobre los origenes}

La Philosophische Praxis llega a Italia en los años noventa. En el 1999, en Turín, tiene lugar la fundación de la primera Associazione Italiana di Counseling Filosofico (AICF), nacida gracias a la comunión de ideas, intenciones y motivaciones de un grupo de 
filósofos, expertos en psicología y de otros sectores disciplinares que han creído, juntos, en las enormes potencialidades de esta nueva disciplina. Cuando la AICF todavía vivía sus primeros compases, al final de 2001, la cooperación entre filósofos y expertos de la psique, que hubiera podido crear un futuro de colaboración y cooperación, fue de repente interrumpida por las exigencias y perspectivas filosóficas y psicológicas de diversas personas del grupo originario.

Allí se presentó, otra vez, el problema del "nombre de la cosa". De este modo, se escinden dos asociaciones: por un lado, la Societa' Italiana Counseling Filosofico (SICoF), que junto a filósofos incluía profesionales de varios sectores interdisciplinares con formación especifica en las Practicas Filosóficas, y, por el otro, Phronesis (Associazione Italiana per la Consulenza Filosofica), compuesta solo por "filósofos" (licenciados y graduados en filosofía).

Desde el principio, fue claro que la diferencia entre Counseling Filosofico y Consulenza Filosofica no era solo una diferencia de nombre, sino que hacia referencia a dos universos teóricos, semánticos, simbólicos y prácticos. Después de quince años, esta escisión originaria entre dos universos asociativos y dos visiones del mundo-disciplina se ha manifestado como favorable al movimiento en Italia: los debates que esta separación ha suscitado en los convenios, en las paginas de las revistas de la respectivas asociaciones y en las publicaciones que veían la luz han permitido desarrollar reflexiones críticas y experiencias prácticas de la disciplina.

Hoy, el debate entre Counseling-Consulenza aun no ha sido superado; sin embargo, ha pasado a ser un tema de segundo nivel. Ahora, hay muchas asociaciones de Prácticas Filosoficas, escuelas de formación y master en las principales universidades italianas y muchos profesionales consultores, counselors, coaches, orientadores, filósofos practicantes, que compartían la idea 
originaria de la Philosophisce Praxis y que la han hecho operativa en los diferentes contextos de aplicación de la disciplina. Aunque esta difusión pueda dificultas, en ocasiones, en torno al nombre de la profesión, el tema esencial reside en la realización de la actividad desarrollada: saber lo que se hace con la cosa-filosofía que vuelve a ser práctica.

Así pues, este discurso sobre el origen puede ayudarnos a aclarar lo que se hace con la filosofía-práctica hoy día.

\section{¿Counseling o Consulenza?}

En el mundo de la vida, las posiciones teóricas son confusas: son borrosas, caóticas, no lineares, pero las dos posiciones típicoideales (Max Weber) del nombre-significado-cosa Consulenza y "Counseling" ayudan a comprender las perspectivas con las cuales se enfrentaron los problemas originarios, por ejemplo, si la filosofía que se vuelve práctica tiene un "método", un "no-método", "un método más allá del método", si y en qué condiciones puede ser entendida como cura o terapia y, en consecuencia, a quienes y por qué puede curar, además, qué instrumentos han de estar presentes en la caja de herramientas del filosofo practicante.

La Philosophische Praxis originaria no era de gran ayuda para orientarse entre estas cuestiones por culpa de la "ontología antinómica" 2 que se manifestaba en la tendencia a definir en términos negativos la nueva disciplina en lugar de aportar indicaciones sobre la identidad, los métodos, las herramientas y la concurrencia a las cuales se hacia referencia.

En los primeros artículos ${ }^{3}$, se lee que la Philosophische Praxis no refleja la filosofía del "gueto académico (...) practicada por los expertos para otros colegas especialistas", "no se ocupa de sistemas

\footnotetext{
${ }^{2}$ RAABE P., Teoria e pratica della consulenza filosofica, Apogeo, Milano, 2006, p. 194

${ }^{3}$ Ahora recopilados en Achenbach G., La consulenza filosofica, Apogeo, Milano, 2004.
} 
filosóficos", "no construye ninguna filosofía" y "no es un adiestramiento filosófico que opera con los patrimonios filosóficos trasmitidos"4. Sin duda, la práctica filosófica no es una filosofía académica, sino que sale del gueto para encontrar a personas en carne y hueso, con sus problemas reales, concretos, existenciales, decisionales, sentimentales, de sentido de los eventos que ocurren, es más aquellas cuestiones que pueden dejar la vida sin sentido, sin amor, sin sabor, sin un cómo ni un porqué.

La filosofía sale del gueto para volver a su antigua vocación de "terapéutica de la condición humana". Actualmente, los problemas de sentido y de significado, y también aquellos existenciales, relacionales, decisionales, sentimentales-emotivos y, en general, de crisis del ser-en-el-mundo se encuentran bajo el monopolio de la psicología-psicoterapia-medicina. La Philosophische Praxis afirma no querer volver a ser una psicoterapia alternativa con respecto a las del mercado de la curación sino una "alternativa a la psicoterapia".

Achenbach sostenía que la filosofía no era en absoluto una cura, no tenía ambiciones terapéuticas, y no debía tener pretender ayudar las personas. Así evitaba confundir la Philosophische Praxis con la psicología y la psicoterapia. Desde este punto de vista, la Philosophische Praxis quería ser un "diálogo libre" entre dos seres humanos que, de manera paritaria, se centran en "poner el pensamiento en movimiento", "vivificar" y "revitalizar" la visión del mundo del consultante. Precisamente, el hecho de ser un "dialogo filosófico libre", exento de todas ambiciones terapéuticas o de cura de la persona, impedía una precisa definición de la nueva disciplina e impedía establecer un método, unas normas o unas fases procedimentales claras en la consulta. Achenbach era bastante categórico al afirmar que "la filosofía no trabaja con los métodos sino sobre los métodos", justificando su afirmación añadiendo que

\footnotetext{
${ }^{4}$ Idem, pp. $69-84$
} 
"la obediencia al método es propia de las ciencias, no de la filosofía" . Todo esto es muy cuestionable.

Su rechazo a ofrecer una definición estándar de la disciplina y, en general, de construir un método y una teoría de la Philosophische Praxis no son más que aspectos particulares de su distanciamiento de la necesidad, típica de las psicoterapias nacida del culto de las ciencias positivas, de normalizar las teorías y las terapias, y además de medir la eficacia de las intervenciones terapéuticas. Puesto que la Philosophische Praxis es, ante todo, una auténtica filosofía caracterizada por una "libertad sin fronteras" que genera "un asombro producido en el dialogo" ha de renunciar a "todas las convicciones seguras de sí mismas" y no debe alzar el problema del método y la eficacia de la ayuda como límite. "La filosofía, de hecho, 'no sabe' donde quiere llegar, pone en duda cualquier criterio de juicio y toda finalidad, por ello debería ser posible hablar en su seno de eficacia" 6 .

La posición de Achenbach tiene algo seductor y muchos filósofos practicantes, no solo en Italia, se han quedado fascinados por la libertad sin fronteras típica de una disciplina que tiene un "método no-metodo" o "método más allá del método" ("beyond method"7), por una profesión que no es una relación de ayuda y que no se cuestiona el problema de la eficacia de su intervención no terapéutica. Otros filósofos practicantes han reaccionado a esta postura negativamente conscientes de que una libertad no reglamentada y sin límites puede abrir la senda a prejuicios y puede ser una "condena por la misma libertad" tanto para el orientador como para el consultante. Peter Raabe, por ejemplo, abrazando la visión del pragmatismo típico anglosajón, es uno de los primeros

\footnotetext{
${ }^{5}$ Idem, p. 13.

${ }^{6}$ RAABE P., op cit., pp. $82-83$

${ }^{7}$ SCHUSTER S., Philosophy Practice: An Alternative to Counseling and Psychotherapy, Praeger Pub, 1999, p. 47.

${ }^{8}$ SARTRE, JP. L'essere e il nulla, Il Saggiatore, Milano, 1943, p. 345
} 
críticos de la versión metodológica del anti o del mas allá propia de Achenbach: si el filosofo practicante asume este planteamiento, escribe, "no se queda en una 'posición objetiva' desde la cual pueda mirar los eventos de la sesión y, por tanto, no dispone de ninguna herramienta con la cual juzgar las consecuencias pragmáticas, en términos de beneficios o perjuicios por el cliente. Esto lleva lógicamente a la conclusión de que cualquier decisión intencional del tema de debate o de la dirección que tomar para sacar adelante el proceso dialógico sea completamente accidental y, de hecho, carente de sentido, ya que, sin los conceptos de progreso y eficacia, cualquier opción tendría la categorización de buena o cualquier otra",

\section{La filosofía encuentra el counseling}

En este punto, se sitúa el encuentro entre las Prácticas Filosoficas y el arte del Counseling, que parece ofrecer la sabiduría, las habilidades y las herramientas necesarias al filósofo aplicado que, salido del gueto académico, encuentra personas verdadera, con problemas reales, y con un bagaje de pensamientos, sentimientos y emociones que manejar. Antes de ilustrar el encuentro de las prácticas de Filosofía Aplicada con el arte del Counseling, clarifiquemos que, no solo en Italia, muchos filósofos practicantes, haciendo referencia a la Philosophische Praxis, sostienen que el "nombre" counseling, ya de por si y a priori, remitiría a una "cosa" híbridamente confundida y mezclada con elementos psicológicos, filosóficos, "orientales" y también New Age. El encuentro entre la "pureza" de la filosofía y el counseling -se afirma- podría generar tipologías de consulta, "técnicas", "estratégicas" e "instrumentos" con una declarada intencionalidad de "ayudar" a las personas. Esta

${ }^{9}$ RAABE P., op. cit., pp 191 y sig. 
"ayuda" ofrecida por el filosofo aplicado haría su tarea más parecida a la del los profesionales de otras relaciones de ayuda y perjudicaría la autenticidad del "hacer" filosófico. En realidad, la filosofía dispondría de todas las competencias necesarias para manejar el encuentro con la persona que necesita consulta filosófica sin necesidad de hacer referencia a los saberes y habilidades de otras disciplinas.

En caso de que sea posible separar los límites entre filosofía y otras disciplinas y saber con precisión qué es lo filosófico y lo psicológico, la cuestión que hoy todavía mueve el debate internacional apunta a cuál es la función del filósofo en relación a los conceptos de "ayudar" o "cuidar". De hecho, siempre que haya una declarada "intención de ayudar" o de ofrecer una "cura" a través de la filosofía, la intervención del filósofo podría entenderse como una forma de psicoterapia camuflada y vacía, de counseling psicológico o de formas integradas como relaciones de cura y de ayuda.

Algunos de los filósofos practicantes son categóricos en esto: evitan referencias a los términos "ayuda", "cura-terapia", "relación" o "escucha activa" al estar demasiado contaminados por la psicología y por los lenguajes "terapéuticos" de la sociedad actual. Entre las tareas de las Prácticas Filosóficas, la aclaración y la depuración del idioma tiene un papel fundamental. Es necesario comprender el significado de los términos "ayudar" y "cuidar", para evitar inútiles equívocos entre lo que hace el filósofo aplicado y el psicólogo-psicoterapeuta. Además, aquí, se corre el riesgo de una paradoja: si el filósofo practicante no entendiera qué es ayudar al individuo y cuidarlo, su profesión sería paradójica, no podría cumplir los requerimientos de su cliente-consultante. El mismo Achenbach, en uno de los primeros ensayos en el cual se enfrentaba con la "cuestión terapéutica"10, defendía que el consultante nunca

${ }^{10}$ ACHENBACH, G., op. cit., pp. 92 y ss. 
solicita la intervención del filosofo practicante para enfrentarse a cuestiones abstractas -como por ejemplo "qué es la felicidad", "cuál es el sentido de la vida", etc.- sino que se presenta siempre con un problema concreto, vital y existencial que ha de resolver cuanto antes. El cliente no pide solo reflexionar sobre la felicidad o plantea sólo cuestiones generales sobre el sentido de la vida, sino que llega siempre con un "relato" de lo que hace su vida infeliz, o sea de los acontecimientos que han vaciado su vida de sentido. Su historia contiene problemas, dilemas, algunas veces misterios, está contaminada de emociones, sentimientos y pasiones, es narrada, a veces, rápidamente $\mathrm{y}$, a veces, con largas pausas de reflexión e incluso con lágrimas.

El consultante necesita la intervención del consultor-siempre y en todo momento- porque vive una situación problemática y porque busca, primariamente, una forma de ayuda para salir de la situación problemática en que se encuentra. En la filosofía encarnada en la práctica, busca, sobre todo, acogida, comprensión y escucha, después, la palabra, la explicación, la advertencia, la revelación; busca una relación y las cualidades humanas del encuentro antes que la sabiduría del filosofo.

Esta conciencia anima a SICoF desde sus inicios. Desde siempre, se ha caracterizado por una mirada interdisciplinar y por un profundo dialogo entre disciplinas filosóficas, psicológicas, médicas y científicas. SICoF se compromete con la construcción del counseling filosófico, una disciplina única, particular y peculiar que reúne en su interior las habilidades, los instrumentos y los métodos del arte del counseling con el vasto patrimonio de las ideas, conceptos, saberes y métodos de investigación de la tradición filosófica. 


\section{El equipaje para el gran salto}

El filósofo aplicado no encuentra libros sino personas, no estudia teorías sino escucha "relatos de vida". El principal salto desde la teoría a la vida verdadera necesita de un equipaje que permita manejar todas las variables del encuentro con el otro. El arte del counseling filosófico nace de la misma exigencia realizada al psicológico: asistir el consultor psicólogo en el "gran salto" desde las teorías de práctica clínica que tiene en la mente, donde no encuentra "casos clínicos", a los "relatos de vida", desde la lectura de las "anámnesis" a las "biografías". Hacer counseling, y en particular Counseling Filosófico, no significa hacer psicología ni psicoterapia, a pesar de existir un "counseling psicológico" y muchas formas y orientaciones híbridas que reúnen saberes de diferente procedencia y cultura que hacen difícil orientarse entre las diferentes practicas terapéuticas.

A nivel internacional, se teme que si el filósofo incluyera en su caja de herramientas habilidades e instrumentos del counseling se arriesgaría a caer en el ámbito psicológico, a invadir el espacio que pertenece a otros y a abrir una acción "terapéutica, técnica, estratégica e instrumental".

Sin duda, el counseling nace, en la segunda mitad del siglo pasado, en el universo psicológico: nace en el seno de la "tercera fuerza en psicología", junto al psicoanálisis y con un sesgo "cognitivo-conductural", que dominaban la escena de la cura de la psique a nivel internacional. El terreno sobre el cual se alza El arte del Counseling de Rollo May es de naturaleza "fenomenológicoexistencial" y se contamina de filosofía y psicología: un terreno bastante fértil para sugerir una relación de ayuda alternativa a las psicologías construidas sobre bases científicas y experimentales. El counseling se presentaba como alternativa al "tecnicismo instrumental y estratégico" de la psicoterapia de la época, como nueva disciplina y también relación de ayuda "centrada en la 
persona-consultante" y no sobre las teorías "científicas" y su consiguientes prácticas psicoterapéuticas.

De esta forma, el counseling no es un esqueleto de técnicas, instrumentos y estrategias psicológicas, sino una unión de habilidades, actitudes y modalidades y técnicas expertise para vivir la relación con quien no soy yo. Rogers era categórico en este punto: lo que hace eficaz el ayuda del Counselor no son solo sus teorías y técnicas, sino las acciones y lo que sucede en la relaciones humanas entre personas que se encuentran. Cuando Rogers hablaba de encuentro, se refería a la relación "yo-tú" de Martin Buber en lugar de la idea de encuentro "clínico" de otras psicoterapias.

La habilidad, de naturaleza comunicativa y relacional, del counselor (la empatía, la escucha activa, la congruencia, la aceptación incondicionada, solo para nombrar las principales), más que ser de carácter psicológico, están presentes en cada persona "dedicada a ayudar al otro"; por ello, están presentes en cada relación humana. Cualquiera, cuando es necesario, pueden ser empático, sabe escuchar, hacer las preguntas correctas en el momento oportuno o aceptar lo que el otro dice sin juzgar su afirmación. El counselor utiliza estas habilidades de manera sistemática. Los fundadores del counseling han descubierto que estas habilidades comunicativas-relacionales, siempre que sean practicadas de forma sistemática, escrupulosamente y con las direcciones humanas y relacionales adecuadas (es decir no con orientaciones psico-técnicas-expertise) crean "el ambiente que facilita la relación", quintaesencia de la disciplina y propedéutica del filosofar.

Esta es la primera lección del counseling para los filósofos, sobre todo para los que no tienen como centro de su práctica la relación de ayuda: si la persona no se siente acogida, comprendida, cuidada, si no se saben manejar los silencios y las lágrimas, los gritos de lamento y las variables no verbales del lenguaje, que son un canal privilegiado de las emociones y sentimientos que 
camuflan credencias y palabras, el riesgo que algo pueda escapar, y a lo mejor también hacer daño, es elevado. La creación del clima oportuno para la relación es el escenario existencial en que tiene lugar el encuentro con el otro y donde se alojará el ambiente de confianza y de apertura que permitirá el despliegue de la sesión filosófica.

En la literatura sobre las prácticas filosóficas, algunos -los "metodólogos"- hablan de la "primera fase" de la relación de counseling como "empática" o de acogimiento. En el encuentro con el otro, se debe dejar todo atrás, con una actitud "focalizada-sobreel-otro" y con un equipaje que permita manejar las variables comunicativo-relacionales que surgen en cada relación humana. Esto convierte al filósofo aplicado en alguien más consciente de lo que está pasando en el encuentro con el otro y le capacita para defenderse de los excesos emotivos, propios y los de su invitado. Las emociones, los sentimientos, el dolor y el sufrimiento, el llorar y el gritar son realidades inherentes a la consulta de la Philosophische Praxis. El filósofo que se vuelve aplicado tiene que enfrentarse con estos problemas de gestión de la relación y de la "cura" por medio de la filosofía.

\section{El filosofo encuentra la "psy"}

Bien, ahora deberíamos preguntamos: ¿hacer todo eso es hacer psicología?, ¿significa esto transformar la practica filosófica en una forma hibrida y camuflada de psicoterapia?

Para algunos, sí. Así lo determinan algunos psicólogos en particular y todos aquellos que retienen términos como "escucha", "cura", "relación" en el feudo de la psicología. Si esto fuera así, el sacerdote, el educador, el médico y el enfermero harían psicología cuando en sus profesiones adoptasen las habilidades del counseling para mejorar la comunicación y la relación con el otro. No 
queremos ahora hacer recurrir a la ironía fácil ni complicar aun mas las cosas, pero hay que reconocer que nos encontramos en una situación paradójica Somos conscientes de que existe una "escucha psicológica y clínica", una "cura-psicoterapia", técnicas para crear empatía (o mejor, el rapport, método para manejar la transferencia y contratransferencia, las proyecciones y las contra-proyecciones), instrumentos (cuasi-)científicos para analizar las emociones, los sentimientos, los vividos, test y experimentos para estudiar cada aspecto de la personalidad humana más o menos perturbada. Sin embargo, aquí "escucha", "cura" o "relación" son "nombres de cosas" especificas dentro del paradigma de la psicología como ciencia. En el momento en que el filósofo -como el educador o el sacerdote- utilizasen test, métodos e instrumentos peculiares de los especialistas Psi, es obvio que se generaría una mezcla poco clara y poco honesta entre saberes y competencias. Sería un "abuso de profesión" puesto que el filósofo imitaría al psicólogo, igual que podría hacer un protésico dental con un odontólogo o un fisioterapeuta que prescribiera fármacos como un médico.

Los fundadores del counseling, y todos los que han participado en el desarrollo de la disciplina-relación de ayuda, son categóricos al afirmar que hacer counseling no significa utilizar los instrumentos, las técnicas y los test de la psicología (más o menos) científica y de las orientaciones de la psicoterapia. El counselor psicológico solo es una orientación específica de una disciplina general.

Cuando Rollo May hablaba del arte del counseling y Carl Rogers del "counseling-no-directivo" o del 'contra-modelo', quisieron tomar la distancia de la psicología científicoexperimental encarnada en un cierto psicoanálisis, en el conductismo y, después, de una dirección cognitivo-conductual. Quien lee los textos fundamentales de la disciplina descubre que nunca se habla de técnicas, test, diagnosis, patología, clínica, sino de actitudes, habilidades humanas, empatía, escucha, visiones del 
mundo, cuestiones de sentido y significado. Esto subraya la distancia entre hacer psicología y counseling, en general, y en particular en relación a la primera y la filosofía. Ítem más: al filósofo aplicado que se encuentra el counseling, no se interesa en utilizar los métodos, los instrumentos y los tests de la psicología científica por el simple motivo de que no los necesita; al contrario, su intervención podrá resultar más eficaz si, y solo si, no utiliza la psicología científica.

El counseling en su dimensión "artística" y la larga tradición filosófica, desde Oriente a Occidente, ofrecen todas las herramientas para curar con la filosofía.

\section{La cura con la filosofía}

¿La filosofía puede curar? reza el título de un libro de Pier Aldo Rovatti. Para contestar a esta pregunta es necesario analizar el significado del termino "cura", en particular en la sociedad de hoy, caracterizada por la medicalización del tejido social y por la transformación del homo sapiens en homo psycologicus. La cura, en este contexto socio-político, es el monopolio del "saber-poder" médico-psi.

Semánticamente, son, por lo menos, tres los significados del termino cura. El primero deriva del latín y se refiere al papel de administrar, manejar, hacerse cargo (por ejemplo, la cura o cuidado de la casa, del negocio). El segundo hace referencia al termino griego therapeia y es un sinónimo de "tratamiento" o "curación". El tercero, finalmente, apunta a la esfera "pasional" o "emotiva" de la cura y se pone en conexión con el sentimiento de preocupación, de ansiedad pero también de solicitud, protección, salvaguardia y atención en relación al sufrimiento de otra persona o a la protección de los débiles (la cura de los enfermos, de los neonatos o los ancianos). Estas etimologías, en el curso de la historia occidental 
más o menos reciente, vienen declinadas según dos modelos. En primer lugar, el "cuidar" (en alemán sorge y en ingles to care) se refiere sobre todo al significado de satisfacción de las necesidades y exigencias e implica el hecho de quedarse en un estado de aprensión, de ansiedad en relación al objeto de nuestras curas. El segundo modelo se refiere al ámbito de la "terapia" y del "tratamiento" médico, sustancialmente dirigido a superar una determinada patología del cuerpo (biòs) o de la mente (psychè) para obtener curación del paciente.

La conexión semántica cura-tratamiento-curación, que lleva a considerar el término cura y los conceptos terapia-tratamiento médico-psi como sinónimos, ha sido suficiente para ofrecer a las disciplinas médico-sanitarias el monopolio de la cura. A pesar de su otro origen semántico griego, esta teoría la afirma solo a partir del nacimiento del la medicina científica moderna y de la "psicología como ciencia".

Para la medicina de siglos precedentes, cuando la psicología todavía no existía como disciplina autónoma sino que formaba parte de la filosofía, no era ni lejanamente pensable la idea de poder curar un cuerpo-psique enfermo sin hacerse cargo de la persona en su totalidad y de sus expresiones y manifestaciones. La cura conservaba el carácter global de "cuidar" al otro por sí mismo, de gestionar una situación patológica de dolor y sufrimiento que afligía al cuerpo y al alma de la víctima de la enfermedad. Esta visión global de cura al cual los filósofos y médicos del pasado se referían ha sido sustituida cuando se distinguieron la medicina, la psicología y la ciencia-técnica experimental, cuando cada malestar fue transformado en enfermedad, cuando la investigación de la causa ha sustituido la búsqueda del significado de la patología, cuando la atención hacia el dato/caso clínico ha sustituido la atención hacia la historia vivida. La cura ha mudado, en este caso, en diagnosis, prognosis, terapia, anamnesis, se ha convertido en terapia-tratamiento de un cuerpo-psique enfermos, disfuncionales o 
a-normales. Si la "cura" se entiende en este sentido, queda claro que la filosofía no puede curar: no cuenta con métodos e instrumentos para hacer diagnosis y prognosis, para intervenir con remedios terapéuticos o para sanar el cuerpo y la psique enferma.

Ahora bien, la cura no solo es esto: la persona que sufre en la sociedad contemporánea no necesita solo esto. Con este discurso no queremos poner en duda o criticar la medicina y la psicología como ciencia. No seria honesto, dado que todos disfrutamos de los beneficios que la evolución de la bio-psico-tecno-medicina ha entregado a la humanidad. No seria correcto tampoco criticar el reduccionismo que la caracteriza, porque la reducción del humano a cuerpo y psique y la cura a terapia-tratamiento ha permitido los citados beneficios. Son intolerables las criticas a la medicinapsicología científica por parte de quien disfruta de ella, sería como criticar a los carnívoros mientras que festejan con una barbacoa de carne.

La medicina y la psicología científica funcionan solo si ejecutan su reducción, si se objetiviza el sujeto, si simplifican lo complejo, si clasifican, investigan causas-efectos y analizan datos. Los mismos médicos y psicólogos son conscientes de esto y, por eso, en el interior de la medicina-psi de la edad de la técnica están naciendo proyectos de Medical Humanities en que las disciplinas bio-tecno-científicas, ciencias humanas y artes adoptan ideas que aspiran a la cura global del paciente considerado como una totalidad bio-psico-social y existencial y no solo como cuerpopsique-maquina defectuoso. Si la cura es global, entonces sí que la filosofía puede cuidar-curar a las personas.

Desde este punto de vista, uno de las tareas más importante de la practica filosófica como cura es "socio-política" antes aun de hablar de counseling. De hecho, como ha dicho Rovatti, la cura con la filosofía empieza desde una dimensión socio-politica: su tarea primaria es intervenir directamente sobre los aparatos, los mundos que generan sufrimiento, antes que sobre las visiones del mundo de 
las personas. El filósofo aplicado tiene la obligación de intervenir en las estructuras sanitarias para proponer proyectos interdisciplinares que asistan a los técnicos del cuerpo y a los técnicos del alma a actualizar la cura global, tiene el deber de intervenir en las escuelas con proyectos dirigidos a hacerse cargo de los pensamientos y de las emociones de los niños y los adolescentes, que no son cabezas vacias que haya que llenar; ha de actuar en las empresas pobladas de "em-pleados", en las cárceles con los detenidos y en todos los lugares de la sociedad donde el sufrimiento no se origina solo a partir del individuo, sino desde el contexto social en que vive. Cada uno de estos mundos tiene en cuenta sus objetivos propios y no la realización individual de los de quienes trabajan en ellos. Por eso, genera formas de "sufrimiento" que no necesariamente son enfermedades. Aquí, el counseling filosófico sigue un trayecto diferente al de la psicología: no interviene sobre el individuo sino sobre el ambiente con la conciencia de que el ser humano es, como dice Heidegger, un seren-el-mundo (in-der-Welt-sein), siendo sobre este mundo sobre el que hay que actuar ${ }^{11}$.

El principal enemigo de las practicas filosóficas, a nivel sociopolitico, es entonces la "medicalización de la condición humana" 12 y el propagarse de la "cura terapéutica" destinada a promover no tanto la autorrealización sino una autoeliminación del individuo; se postula un sí mismo frágil y débil y se llega a creer que, para manejar la existencia, sea necesario un continuo recurso a prácticas-tratamientos terapéuticos, hasta el punto de encontrar alivio en una diagnosis y en una "terapia empapada de fármacos". El counseling filosófico, que no hace diagnosis porque no cura

\footnotetext{
${ }^{11}$ GALIMBERTI U., "Presentazione", Dizionario del counseling filosofico e delle pratiche filosofiche (a cura di NAVE. L., PONTREMOLI P., ZAMARCHI E.), Mimesis, Milano, 2013.

12 FRANK Furedy, Therapy Culture: Cultivating Vulnerability in an Uncertain Age, Routeledge, New York, 2004, p. 101.
} 
enfermedades, está de acuerdo con Furedi en creer que la cultura terapéutica acaba determinando una especie de control social con el que "enseñar a quedarse en su puesto, ofreciendo a cambio las dudas benéficas de la confirmación y del reconocimiento social".

La tarea social y política de una cura con la filosofía no se queda, en Italia, en ser un ideal programático. Se atestigua la existencia de cientos de proyectos realizados por los filósofos practicantes en las escuelas, los hospitales, las empresas y en otros contextos en que la filosofía, platónicamente, puede ser puesta a servicio del ser-en-el-mundo.

En suma, la cura en el contexto socio-político de la filosofía no tiene nada de psicológico. Al contrario, con Rovatti, reiteramos que su enemigo principal es la cura medicalizada y psi que transforma el malestar en enfermedad del bios o de la psichè, con consecuente sometimiento del paciente a las terapias del médico o del psicólogo.

Vayamos más allá, en el encuentro con el individuo o con el grupo, el couselor filosófico - y el filósofo aplicado en general¿necesita filosofía? Después de haber esclarecido el significado del counseling y del término cura, podemos concluir que el counseling filosófico proporciona una cura con filosofía - una terapéutica de la condición humana- absolutamente irreducible a la terapia de la psicología. No disponiendo de un saber diagnóstico ni de técnicas terapéuticas de tratamiento, la cura prevista del counseling filosófico -en línea con el significado ampliado del "cuidar"- se refiere más bien al servicio ofrecido por el filósofo counselor hacia un individuo o grupo con objeto de asistir y acompañar la investigación de sus pensamientos, creencias, valores, necesidades, sentimientos y emociones (de su visión del mundo). En ellos, están íntimamente involucrados y son determinantes los aspectos filosóficos problemáticos de su existencia y quedan implicados capacidades, instrumentos y métodos del counseling con los de la tradición filosófica, se establece una relación de ayuda de 
naturaleza hermenéutica y empática que facilita en el consultante el conocimiento y la cura de sí mismo.

El alma del counseling y el alma del filósofo, que caracterizan esta relación de ayuda de orientación filosófica, se unifican y amalgaman en una única actitud filosófica y empática, que incluya a su interior tanto las habilidades del counseling indispensables para crear "el ambiente que facilita la relación"13 y para prestar atención a lo que pasa al interior de una relación con el otro, para indagar y aclarar las cuestiones problemáticas del counseling.

\section{La actitud filosófica: "saber ser" y "saber hacer"}

La actitud filosófica ${ }^{14}$, en las prácticas filosóficas en particular, indica ante todo una cierta manera de ser: es un saber ser antes que saber hacer. Es la inclinación que caracteriza quien piensa de forma filosófica respeto a quien no lo hace, de quien sabe y sabe ayudar los otros a tener alto el sentido de la admiración, del estupor frente de las cosas junto a la capacidad de dejarse sorprender, motivo por el cual la filosofía nación ${ }^{15}$. La actitud filosófica la tiene quien contempla y favorece la contemplación de nuevas posibilidades, imagina nuevos escenarios, somete a crítica las creencias ejercitando y desarrollando un pensamiento crítico por medio de procesos mentales de discernimiento, análisis y evaluación para ir más allá de la parcialidad del individuo. Es un tutelaje contra el engaño, la superstición y el desconocimiento de sí mismo y del mundo, que nos vuelve conscientes de la imposibilidad de llegar a una absoluta certidumbre, a un conocimiento exacto y a una universalidad ineludible. El filósofo en el encuentro con el otro

\footnotetext{
${ }^{13}$ ROGERS Carl, La terapia centrata sul cliente, La nuova Italia, Firenze, 1999, 64.

${ }^{14}$ PONTREMOLI P., NAVE L., "Atteggiamento Filosofico" in Dizionario, op. cit., pp. 102 y sig.

${ }^{15}$ Cfr. ARISTÓTELES, Metafísica, 982b-983a.
} 
cuestiona lo incuestionable, hace preguntas sobre lo que no se sabe, sobre lo que cree saber pero que, bajo de una mirada crítica, esconde mucho más que lo que se piensa y se dice.

Uno de los métodos privilegiados es el método mayéutico socrático: se ayuda al consultante a dar a la luz los frutos de su propia visión del mundo y se cuidan sus pensamientos, creencias, valores morales y la vida emocional que forman parte de su historia. Si los tests, el rapport o la transferencia pertenecen a la psicología como ciencia y a la psicoterapia clínica, el método mayéutico y el dialogo socrático son métodos filosóficos. Sin duda, los psicólogos y otros profesionales pueden utilizar la mayéutica para "dar a la luz pensamientos", pero el filósofo es consciente de que la mayéutica no es solo esto. En el contexto de las prácticas filosóficas, la mayéutica presenta fundamentos "socráticos" concretos (que no podemos tratar aquí) mientras que el Dialogo Socrático posee una metodología compartida a nivel internacional y que solo el profesional experto en Filosofía Aplicada sabe practicar. Éste es el motivo por el que hacer filosofía práctica y hacer psicoterapia no es lo mismo. Junto a la Mayéutica y al Dialogo Socrático, en la caja de herramientas del filosofo practicante, hay muchas otras herramientas, como el "método argumentativo", el "método hermenéutico" o el "método crítico", que hacen el trabajo del filósofo original en relación a las otras profesiones de ayuda.

El filósofo aplicado, y no el psicólogo, pone la filosofía al servicio del ser-humano-en-el-mundo, incluidos los que no saben mucho de filosofía. Ponen a disposición el vasto patrimonio histórico de teorías, conceptos, maneras de indagar, reflexionar y argumentar de modo que cada persona pueda aprovechar lo que, tal vez, no conozca bien. La Filosofía Aplicada no acaba de ser un lugar donde se hacen preguntas y se plantean dudas, según su naturaleza crítico-inquisitiva, que también comparte con otras disciplinas, sino que se configura como una práctica 
transformadora en que el pensamiento, el pensante y el pensado se auto-determinan y se transfiguran continuamente $y$ en manera recíproca.

La actitud filosófica representa entonces uno de los fundamentos del counseling filosófico: en la mesura con que el profesional, en el encuentro con el consultante, crea una relación de ayuda activa, comprensiva y empática, direccionada hacia la búsqueda filosófica de su visión del mundo y su sistema de creencias con el objetivo de comprender los pensamientos, los valores, las pre-comprensiones y las modalidades de reaccionar emotivamente a los eventos, sobre todo, donde se crea una discrepancia entre sus visión del mundo y el mundo objeto de su visión. El filósofo aplicado se basa en los hechos y las informaciones presentes en el encuentro para conducir al consultante más allá de estos datos y estos hechos, al fin de problematizar la peculiar modalidad de pensar y de vivir las situaciones existenciales que generan insatisfacción, sufrimiento, malestar. El counselor filosófico asume una auténtica actitud filosófica y no prescribe remedios fáciles y pastillas de sabiduría a la persona que sufre y proporciona un clima empático que facilita la relación, va a la búsqueda de los aspectos problemáticos de la visión del mundo de la persona que generan sufrimiento y malestar: incoherencias o tensiones entre dos modalidad existenciales a través de las cuales la vida debe ser vivida, presupuestos no examinados y tomados a-críticamente, expectativas que no pueden ser satisfechas realistamente, errores de perspectiva y de evaluación del comportamiento, sobregeneralizaciones, imposiciones del deber, catastrofismos que contribuyen a generar o prolongar emociones malsanas y el malestar existencial. La actitud filosófica constituye una actitud crítica que insta a indagar en las ideas, los conceptos, los valores que guían, de manera mas o menos consciente, la vida de la persona, a descubrir presupuestos implícitos, a descubrir las incoherencias y las estructuras 
escondidas que rigen un proyecto existencial, estimulando la sensibilidad argumentativa.

El fillosofo, en el curso del counseling individual o durante las sesiones de las diferentes practicas filosóficas, anima al consultante a adoptar una actitud filosófica, a tomar conciencia y a cuestionar su propio punto de vista sobre el mundo argumentándolo en frente de posibles contra-argumentaciones del interlocutor. Así, mostrará y ganará una visión mas compleja de la situación problemática o del argumento objeto de investigación, actualizando un cambio de perspectiva o un descentramiento existencial que tiene lugar en el contexto de un auténtico diálogo, entendido como intercambio de razones y contra-razones a la investigación de la serenidad, la verdad y el bienestar.

La practica filosófica entonces puede tener un valor curativo de la condición humana, sobre la base de la mesura que permite a los individuos acceder a un mayor auto-conocimiento, conciencia de su propia visión del mundo, de comprender el sentido de los hechos y de las situaciones en que tiene lugar su vida en el mundo, así como a clarificar y activar su proyecto existencial, que según los antiguos filósofos era conditio sine qua non de cada un auténtico bienestar global o Eudaimonia.

\section{Conclusión}

Volvemos, en la conclusión, al problema del nombre de la cosa que, como dijimos, parecía un problema superado. En Italia, hay muchos nombres para cualificar la filosofía que ha abandonado el gueto académico y existen muchas realidades asociativas, escuelas y profesionales que utilizan "nombres" y hacen "cosas" diferentes a lo realizado en la universidad. Quizás, sea el momento de realizar un censo de todos esos "nombres" y de todas esas "cosas" presentes en territorio nacional. 
Aun cuando el nombre de la disciplina no sea connotativo, en este punto, lo más urgente es reflexionar sobre lo que se está haciendo, sobre los instrumentos, métodos, saberes y prácticas que se disponen y sobre cuál es el objetivo de la practica filosófica: el bienestar de los consultantes que requieren el ayuda del filosofo para enfrentarse a una cuestión de su existencia. Los problemas son de naturaleza epistemológica y ética: por un lado es necesario reflexionar sobre los saberes, métodos e instrumentos para el encuentro con el otro, que pueden proceder de la tradición filosófica y de otros sectores disciplinares. En este estudio, hemos privilegiado el encuentro entre filosofía y arte del counseling, pero en Italia las vinculaciones interdisciplinares son muchas: el coaching, la Programación Neuro-Lingüística, la Pet-Therapy, el training autógeno, la sabiduría del I Ching, la tradición de los "ejercicios espirituales" y otras prácticas de Oriente y Occidente. El debate epistemológico es importante para la cuestión ética y deontológica que se refiere a lo que acontece en la persona que busca la ayuda del filósofo para contestar a un problema específico de su vida. Aquí está en juego la responsabilidad, la honestad y el respeto hacia el otro y hacia uno mismo.

Si el filosofo aplicado cuenta con una caja de herramientas con los conocimientos, los métodos, los instrumentos y las prácticas para satisfacer esta necesidad sabrá materializar su profesión y respetar el ética y la deontología profesional, el resto pueden gentilmente volverse al gueto académico desde el que han salido torpemente. 


\section{Bibliografía}

ACHENBACH G., La consulenza filosofica, Apogeo, Milano 2004.

FUREDY F., Therapy Culture: Cultivating Vulnerability in an Uncertain Age, Routeledge, New York, 2004, p. 101

LAHAV R., Comprendere la vita, Apogeo, Milano, 2004.

MAY R., L'arte del counseling, Astrolabio, Milano, 2002.

NAVE L., BISOLLO M., Filosofia del benessere. La cura dei pensieri e delle emozioni, Mimesis Edizioni, Milano, 2010.

NAVE L., PONTREMOLI P., ZAMARCHI E. (a cura di), Dizionario del Counseling Filosofico e delle Pratiche filosofiche, Mimesis, Milano, 2013.

RAABE P., Teoria e pratica della consulenza filosofica, Apogeo, Milano, 2006.

ROGERS C., La terapia centrata sul cliente, La nuova Italia, Firenze, 1999.

ROVATTI A., La filosofia può curare?, Raffaello Cortina, Milano 2006.

SARTRE J.P., L'essere e il nulla, Il Saggiatore, Milano, 1943.

SCHUSTER S., Philosophy Practice: An Alternative to Counseling and Psychotherapy, Praeger Pub, 1999.

ZAMARCHI E., CONTESINI S., Sensibilità filosofica, Apogeo, Milano 2010. 